\section{Perfil epidemiológico e clínico de pacientes admitidas com diagnóstico de sepse puerperal de origem pélvica em uma UTI obstétrica no Nordeste do Brasil}

\section{Epidemiological and clinical profile of patients admitted at an obstetrical ICU with diagnosis of puerperal pelvic sepsis in Northeastern Brazil}

\section{Abstract}

Objectives: to describe the clinical and epidemiological profile of patients admitted with the diagnosis of puerperal sepsis of pelvic origin in an intensive care unit (ICU).

Methods: a cross-sectional study, from February to August 2010 was conducted. Cases of puerperal sepsis from pelvic origin admitted to the ICU obstetric Institute of Integrative Medicine Professor Fernando Figueira (IMIP) - city of Recife - Brazil were investigated. The variables were: age, origin, conducting pre-natal, delivery route, use of urinary catheter, central venous catheter, intubation, vasoactive drugs, conducting relaparotomy, the number of performed relaparotomies, performing hysterectomies, the incidence of complications and death.

Results: 77 identified admissions for sepsis, and 35 postpartum. The mean age was 22.6 years, most coming from towns of the state. $52.9 \%$ of women were aged 20 years. $62.5 \%$ were primiparous and $68.6 \%$ had undergone cesarean section. Concerning temperature, $42.8 \%$ of patients had values below $35^{\circ} \mathrm{C}$ or above $37.8^{\circ} \mathrm{C}$. Complications occurred in $45.7 \%$ of patients. Dialysis was indicated in $40 \%$ and vasoactive drug used in $22.9 \%$. Hysterectomy was performed in $44.1 \%$ of patients, in $54.3 \%$ relaparotomy necessary.

Conclusions: genital puerperal sepsis is a serious disease that affects young women with low parity. The frequency of complications and invasive procedures in this group of women is high, which implies a high morbidity and mortality. Describing this group of patients contributes with current knowledge about the disease, improving the preparation of the Centers for dealing with genital puerperal sepsis.

Key words Sepsis, Puerperal infection, Cesarean section
Euclides Dias Martins Filho 1

Alan Chaves Dos Santos 2

Renato Stênio Torres Rodrigues Junior 2

Luis Adeodato 3

Isabela Coutinho 4

Leila Katz 5

1,2 Faculdade Pernambucana de Saúde. Rua Jean-Émile Favre, 422. Imbiribeira. Recife, PE, Brasil. CEP: 51200-060

E-mail: euclides@fps.edu.br

3 Universidade de Pernambuco.Recife, PE, Brasil.

4,5 Instituto de Medicina Integral Prof. Fernando Figueira. Recife, PE, Brasil.

\section{Resumo}

Objetivos: descrever o perfil epidemiológico e clínico das pacientes admitidas com o diagnóstico de sepse puerperal de origem pélvica em uma Unidade de terapia intensiva (UTI) obstétrica.

Métodos: um estudo de corte transversal, de fevereiro a agosto de 2010, foi conduzido. Foram investigados os casos de sepse puerperal de origem pélvica admitidas na UTI obstétrica do Instituto de Medicina Integral Prof. Fernando Figueira (IMIP) Recife-Brasil. As variáveis analisadas foram: idade, procedência, realização de pré-natal, via de parto, uso de sonda vesical, uso de cateter venoso central, intubação, uso de droga vasoativa, realização de relaparotomia, o número de relaparotomias realizadas, realização de histerectomia, ocorrência de complicações e óbito.

Resultados: identificamos 77 admissões por sepse, sendo 35 puerperal de origem genital. A idade média foi de 22,6 anos, a maioria procedente de cidades do interior do estado. 52,9\% das mulheres tinham até 20 anos. 62,5\% eram primíparas e 68,6\% haviam sido submetidas a cesárea. Em relação à temperatura, 42,8\% das pacientes apresentaram valores abaixo de $35^{\circ} \mathrm{C}$ ou acima de $37,8^{\circ} \mathrm{C}$. Complicações ocorreram em 45,7\% das pacientes. Diálise foi indicada em $40 \%$ e droga vasoativa utilizada em 22,9\%. Histerectomia foi realizada em 44,1\% das pacientes, sendo necessário relaparotomia em $54,3 \%$.

Conclusões: a sepse puerperal de origem genital é doença grave, que acomete mulheres jovens de baixa paridade. A freqüência de complicações e de procedimentos invasivos nesse grupo de mulheres é alto o que implica em alta morbidade e mortalidade. Conhecer de forma mais detalhada esse grupo de pacientes contribui com o conhecimento atual sobre a doença, melhorando a preparação dos centros para lidar a sepse puerperal de origem genital.

Palavras-chave Sepse, Infecção puerperal, Cesariana 


\section{Introdução}

A sepse é atualmente a principal causa de morte nas unidades de terapia intensiva (UTI). ${ }^{1}$ A taxa das internações hospitalares e nas UTI ocasionadas por esta doença é de aproximadamente $2 \%$ a $11 \%$, e está entre as principais causas de morte nos EUA, com mortalidade entre $20 \%$ e $80 \% .2,3$ As bactérias mais freqüentes observadas em culturas de sangue e outras secreções obtidas de pacientes com sepse são as enterobacterias, os estafilococos e a Pseudomonas aeruginosa. 4

A sepse também é causa importante de morte materna no mundo. Pouco se conhece sobre a real incidência de morbidade grave relacionada à maternidade. É estimado que a sepse puerperal é responsável por 75.000 óbitos/ano, principalmente em países pouco desenvolvidos, com incidência de 0,1 0,6 por 1.000 partos. 5 A infecção puerperal é um termo geral usado para descrever qualquer infecção bacteriana pélvica após o parto. Na grande maioria dos casos, as bactérias responsáveis pela infecção são aquelas que normalmente residem no intestino e também colonizam o períneo, a vagina e a cérvice. 6

A sepse puerperal, quando não conduz à morte, pode causar problemas como a doença inflamatória pélvica (DIP) e infertilidade. 7 Para o diagnóstico de sepse puerperal é recomendada a obtenção de pelo menos duas hemoculturas, sendo uma amostra de sangue periférico e outra proveniente de sangue colhido em veia central, exceto se o cateter tiver sido inserido há menos de 48 horas. Também devem ser realizadas culturas de prováveis sítios infecciosos como urina, liquor, secreções do trato respiratório e pontas de cateteres, entre outros, antes do início da antibioticoterapia.7,8 A etiologia é geralmente polimicrobiana, estando o estreptococo beta-hemolítico do grupo A frequentemente envolvido. O fator de risco imediato mais importante parece ser a cesariana, sendo a antibioticoprofilaxia etapa importante para redução desse risco. ${ }^{5}$

O local mais comum de infecção, na sepse puerperal, é o sítio de inserção da placenta, mas outros locais também freqüentes são as feridas abdominais ou perineais resultantes de cirurgia ou de lacerações do trato genital. ${ }^{9}$ Por outro lado, existem diversos fatores de risco para o desenvolvimento da sepse puerperal identificados no pré-natal e na assistência obstétrica como cesárea, ruptura prematura das membranas, trabalho de parto prolongado, múltiplos exames cervicais e monitorização fetal interna. 6

Há evidências, embora não conclusivas, de que a condição socioeconômica desfavorável e a anemia aumentam a probabilidade de infecção. ${ }^{9} \mathrm{O}$ papel da nutrição na gênese da infecção ainda é discutido, mas há indicações de que a imunidade celular está comprometida em modelos animais de desnutrição. ${ }^{9}$

O caráter multifatorial das complicações e da mortalidade materna torna difícil seu controle. Para redução da morbidade e mortalidade materna se faz necessário conhecer os fatores que acarretam o desenvolvimento da sepse puerperal e, a partir daí, sugerir recomendações e intervenções. 10

No Nordeste do Brasil, onde condições sócioeconômicas desfavoráveis ainda contribuem para uma inadequada assistência ao parto e puerpério, principalmente em cidades do interior, infecções graves durante esse período continuam prevalentes. ${ }^{11}$

No Recife, uma das maiores cidade do nordeste, a UTI obstétrica do Instituto de Medicina Integral Prof. Fernando Figueira (IMIP) é hoje referência no Estado e na região em assistência a puérperas de alto risco e com complicações decorrentes do parto. Dentre as doenças que justificam a admissão na UTI, a sepse puerperal representa um grupo com elevada taxa de complicações e óbito. Neste contexto, o presente estudo aborda um tema de extrema relevância regional e nacional.

\section{Métodos}

Realizamos um estudo de corte transversal com o objetivo de investigar os casos de sepse puerperal entre setembro de 2002 e dezembro de 2009 no IMIP. Para isto foram analisados as pacientes admitidas na UTI obstétrica com diagnóstico de sepse puerperal, 35 das quais tendo sua origem pélvica. A UTI Obstétrica do (IMIP) recebe mensalmente cerca de 80 mulheres durante o período grávido-puerperal criticamente doentes.

Foram excluídas aquelas cujo prontuário tinha informações incongruentes ou incompletas. Foram analisados as seguintes variáveis: idade, procedência, realização de pré-natal, via de parto, retenção placentária, uso de sonda vesical, uso de cateter venoso central, intubação, uso de droga vasoativa, realização de relaparotomia, o número de relaparotomias realizadas, realização de histerectomia, ocorrência de complicações (insuficiência renal, insuficiência respiratória e insuficiência circulatória) e óbito.

No processamento dos dados foi utilizado o programa Epi-Info 3.5.1. Os dados foram apresentados como distribuição de freqüência para as variáveis categóricas, e valores médios para as variáveis quantitativas.

A pesquisa foi aprovada pelo Comitê de Ética em 
Pesquisa (CEP) do IMIP sob o número 1618. Para o presente estudo utilizamos os casos colhidos de forma retrospectiva de um estudo de coorte ainda em andamento.

\section{Resultados}

Durante o período do estudo foram admitidas na UTI 6307 mulheres. Dentre estas, 77 apresentavam sepse puerperal na admissão $(1,2 \%)$ sendo $35(0,55 \%$ do total de admissões no período) com foco genital.
Estas 35 mulheres tinham idade média de 22,6 anos $(\mathrm{dp}=5,6)$ e pouco mais de metade $(53 \%)$, tinha menos de 20 anos. Apenas nove haviam parido na instituição. A mediana de gestações foi de um, variando de uma a oito. Primiparidade foi observada em 22 , o que representa $62,5 \%$ da amostra.

A grande maioria era procedente de cidades do interior do estado $(62,9 \%)$ e em relação à via de parto, detectamos que a cesariana representou $68,6 \%$ do total (Tabela 1).

Tabela 1

Características das mulheres admitidas na UTI Obstétrica do Centro de Atenção a Mulher - Instituto de Medicina Integral Prof Fernando Figueira com diagnóstico de sepse puerperal de origem genital de 2002 a 2009.

\begin{tabular}{lcc}
\hline Características & N & $\%$ \\
\hline Procedência & 13 & 37,1 \\
Recife e região metropolitana & 22 & 62,9 \\
Cidades do interior & 9 & 25,7 \\
Parto no IMIP & 26 & 74,3 \\
Parto fora do IMIP & & \\
Via de parto & 24 & 68,6 \\
Cesárea & 11 & 31,4 \\
Normal & & \\
\hline
\end{tabular}

As características clínicas e laboratoriais no momento da admissão estão mostradas na Tabela 2. Foi observado ainda frequência cardíaca acima de 90 bpm (batimentos por minuto) e frequência respiratória acima de 24 irpm (incursões respiratórias por minuto) em 32 pacientes $(91,4 \%)$ e 22 pacientes $(62,9 \%)$ respectivamente. Em relação à temperatura, 15 pacientes $(42,8 \%)$ apresentaram valores abaixo de $35^{\circ} \mathrm{C}$ ou acima de $37,8^{\circ} \mathrm{C}$. Leucometria acima de $20.000 / \mathrm{mm} 3$ esteve presente em apenas 11 pacientes $(32,4 \%)$; por outro lado o desvio a esquerda esteve presente em $85,7 \%$. Plaquetopenia por sua vez foi registrada em $14(40,6 \%)$ dos casos.

A ocorrência de qualquer complicação, esteve presente em 16 pacientes $(45,7 \%)$, sendo as mais frequentes choque séptico e insuficiência renal, encontrados em $10(28,6 \%)$ e $6(17,1 \%)$ pacientes, respectivamente (Tabela 3 ).

Sonda vesical foi utilizada em $30(85,7 \%)$ dos casos, cateter venoso central em $14(40 \%)$ das pacientes e suporte ventilatório invasivo foi indicado em 5 (42,9\%). A diálise foi indicada em $14(40 \%)$ e droga vasoativa utilizada em $8(22,9 \%)$. A histerectomia foi indicada em 15 pacientes $(44,1 \%)$, sendo necessário relaparotomia em 19 (54,3\%) (Tabela 4). Em relação ao número de relaparotomias, 11 necessitaram de uma re-abordagem após o procedimento cirúrgico inicial, seis mulheres precisaram de duas e duas pacientes de três re-abordagens. O internamento hospitalar variou de um a 55 dias com mediana de oito dias. 
Tabela 2

Características clínicas e laboratoriais das mulheres na admissão na UTI Obstétrica do Centro de Atenção a Mulher Instituto de Medicina Integral Prof Fernando Figueira com diagnóstico de sepse puerperal de origem genital no período de 2002 a 2009.

\begin{tabular}{lrcc}
\hline Características & $\overline{\mathbf{X}} \pm$ DP & Mediana & Min - Máx \\
\hline Frequência Cardíaca (bpm) & $119,5 \pm 25$ & 116 & $56-178$ \\
Frequência Respiratória (irpm) & $31 \pm 13,2$ & 28 & $17-70$ \\
Temperatura $\left({ }^{\circ} \mathrm{C}\right)$ & $36,7 \pm 1,6$ & 36,8 & $33-39,8$ \\
Contagem leucocitária (/mm3) & $17.311 \pm 9.737$ & 13.150 & $4.150-41.780$ \\
\hline
\end{tabular}

Tabela 3

Complicações das mulheres admitidas na UTI Obstétrica do Centro de Atenção a Mulher - Instituto de Medicina Integral Prof Fernando Figueira com diagnóstico de sepse puerperal de origem genital de 2002 a 2009.

\begin{tabular}{lrr}
\hline Complicações & N & $\%$ \\
\hline Qualquer & 16 & 45,7 \\
CIVD* & 2 & 5,7 \\
Insuficiência hepática & 5 & 14,3 \\
Insuficiência renal & 6 & 17,1 \\
Choque séptico & 10 & 28,6 \\
Óbito & 5 & 14,3 \\
\hline
\end{tabular}

${ }^{*} \mathrm{CIVD}=$ coagulação intravascular disseminada.

Tabela 4

Procedimentos mulheres admitidas na UTI Obstétrica do Centro de Atenção a Mulher - Instituto de Medicina Integral Prof Fernando Figueira com diagnóstico de sepse puerperal de origem genital no período de 2002 a 2009.

\begin{tabular}{lrc}
\hline Procedimentos & N & $\%$ \\
\hline Sonda vesical & 30 & 85,7 \\
Cateter venoso central & 14 & 40,0 \\
Suporte ventilatório invasivo & 15 & 42,9 \\
Diálise & 14 & 40,0 \\
Droga vasoativa & 8 & 22,9 \\
Histerectomia & 15 & 44,1 \\
Relaparotomias & 19 & 54,3
\end{tabular}




\section{Discussão}

No presente estudo a sepse puerperal de origem pélvica representou $45 \%$ dos casos de sepse admitidas na UTI obstétrica, o que corresponde a $0,5 \%$ das admissões na unidade.

Alguns autores quantificam a incidência de sepse puerperal de acordo com o numero de partos ocorridos, como Dare em 1998,10 que encontrou 1,7\% de sepse puerperal em 8428 partos avaliados. Contudo, não é adequada a realização de uma análise considerando o número de nascimentos ocorridos neste serviço em relação a frequência de sepse, uma vez que apenas $25 \%$ das mulheres com esse quadro haviam tido partos no mesmo. A baixa frequência aqui descrita pode ser explicada por dois fatores principais. Em primeiro lugar, a população admitida na UTI obstétrica do IMIP é representada em sua maioria (87\%) por mulheres portadoras de distúrbios hipertensivos, mas apenas $2,1 \%$ de mulheres com infecções. ${ }^{11}$ Além disso foram incluídas neste estudo somente mulheres que já na admissão tinham sido classificadas como portadoras de sepse, existindo a possibilidade de que aquelas admitidas com outros diagnósticos tenham posteriormente evoluído com o quadro sem serem identificadas. Essa foi uma limitação causada pelo desenho do estudo.

A taxa de infecção puerperal varia na literatura de $1,2 \%$ a $2,9 \%$. Têm-se descrito um risco três a cinco vezes maior de sepse puerperal após cesarianas quando comparados aos partos normais. ${ }^{5,12-14}$ Este é um achado condizente com o nosso, visto que no grupo que evoluiu com sepse puerperal de origem genital, a via de parto mais frequente foi a abdominal.

Dados sobre a avaliação do perfil das mulheres que evoluem com sepse puerperal são escassos, em virtude de que poucos serviços reúnem número suficiente de pacientes para uma análise. $O$ fato de nosso serviço ser referência para todo o estado de Pernambuco para mulheres no ciclo grávido-puerperal criticamente enfermas permite encontrar uma quantidade considerável de casos dessa doença.

Nossa amostra apresentou uma média de idade de 22,6 anos, coincidindo com a idade média descrita em outro estudo avaliando mortes maternas por todas as causas e no qual a sepse puerperal foi responsável por $13 \%$ dos óbitos. 12 Esta idade coincide ainda com a idade média das pacientes admitidas em nossa unidade de terapia intensiva. ${ }^{11}$

Acompanhando o perfil das pacientes internadas na UTI obstétrica, ${ }^{11}$ a maioria das mulheres é procedente de cidades do interior do estado. Já é reconhecido como fator de risco a transferência de mulheres oriundas de unidades de saúde periféricas para centros terciários com a presença de condições de gravidade em pacientes obstétricas e internamento em unidades de terapia intensiva. 15

Diferentemente dos achados de Amorim et al. 11 em 2006, encontramos uma predominância de primíparas na amostra. Entretanto os nossos achados coincidem com dados obtidos em 2008, em um estudo avaliando o perfil de mulheres admitidas em nesta UTI com critérios de morbidade materna grave. ${ }^{16}$ Chama a atenção a idade precoce de nossas pacientes e da sua baixa paridade, o que implica em grave morbidade numa idade bastante jovem, a qual, embora não levando ao óbito, pode acarretar prejuízos à vida dessas mulheres tanto na esfera reprodutiva, quanto na psicológica e social.

São conhecidos os critérios diagnósticos de sepse na população geral. A aplicação desses critérios em gestantes deve ser feita de forma cuidadosa e com senso critico. As alterações fisiológicas da gestação no organismo materno podem prejudicar no diagnostico. As gestantes podem apresentar taquipnéia e taquicardia, sem obrigatoriamente significar doença.

Apesar da taquicardia presente na maior parte da amostra no momento da admissão, observamos que a taquipnéia e as alterações de temperatura foram menos frequentes. Chamamos a atenção para o fato de que aqui, descrevemos a clinica das mulheres de forma pontual, não sendo é possível assim excluir a possibilidade de que em outros momentos da doença as alterações poderiam estar presentes. Além disso, como a maioria das mulheres era oriunda de outros serviços, é provável que pudessem já ter passado por procedimentos ou inicio de terapêutica capazes de influenciar esse achado.

O leucograma alterado, que também é critério diagnóstico de Síndrome da Resposta Inflamatória Sistêmica (SIRS), é um dado que deve ser avaliado com cautela em puérperas. Sabe-se que este grupo de mulheres, devido as alterações fisiológicas normais da gestação, cursa com leucocitose o que também pode dificultar o diagnóstico de sepse. A presença de formas jovens no leucograma, no entanto, é mais fidedigna e pode servir como um guia para considerarmos o exame como alterado. Em nossa amostra, $85,7 \%$ das mulheres apresentavam desvio á esquerda.

Pelo menos uma complicação aconteceu em $45,7 \%$ das pacientes, sendo as mais frequentes o choque séptico e a insuficiência renal. Choque séptico foi encontrado em $28,6 \%$ das mulheres com diagnóstico de sepse na admissão, o que é uma frequência semelhante àquela descrita em outro 
hospital brasileiro numa UTI geral. 17

Não encontramos estudos descrevendo de forma específica a presença de complicações nesse grupo de mulheres. Realizando comparações com o público atendido na mesma UTI 11,16 aqui estudada, observamos que a frequência de complicações é maior entre as mulheres com sepse puerperal de origem genital do que nas outras formas. Este fato pode ser explicado pelo fato de que uma parcela significativa de nosso público é constituído de mulheres com distúrbios hipertensivos, que permanecem na UTI durante a terapia profilática com sulfato de magnésio e que às vezes complicam gravemente.

Das 35 mulheres aqui estudadas, $14,7 \%$ evoluíram para óbito. Comparando com a frequência de óbito encontrada em pacientes adultos com sepse, essa é uma taxa relativamente baixa, uma vez que na população geral, a taxa de mortalidade é em torno de $30 \% .17,18$

Alguns fatores têm sido sugeridos como responsáveis pelo melhor prognóstico da sepse em obstetrícia: melhor condição clínica da paciente (geralmente jovem e sem comorbidades), sítio de infecção passível de intervenção cirúrgica e maior sensibilidade dos principais microorganismos à antibioticoterapia de amplo espectro. ${ }^{19}$ Frequências

\section{Referências}

1. Martin GS, Mannino DM, Eaton S, Moss M. Epidemiology of sepsis in the United States from 1979 through 2000. N Engl J Med. 2003; 348: 1546-54.

2. Angus DC, Wax RS. Epidemiology of sepsis: an update. Crit Care Med. 2001; 29 (Suppl. 7): S109-16.

3. Friedman G, Silva E, Vincent JL. Has the mortality of septic shock chan $\neg$ ged with time? Crit Care Med. 1998; 26: 2078 86.

4. Veronesi R. Tratado de Infectologia. 2 ed. São Paulo: Atheneu; 2004. p.886-904.

5. Van Dillen J, Zwart J, Schutte J, van Roosmalen J. Maternal sepsis: epidemiology, etiology and outcome. Curr Opin Infect Dis. 2010; 23: 249-54.

6. Lydon-Rochelle M, Holt VL, Martin DP, Easterling TR Association between method of delivery and maternal rehospitalization. J Am Med Assoc. 2000; 283: 2411-16.

7. Dellinger RP, Levy MM, Carlet JM, Bion J, Parker MM, Jaeschke R, et al. Surviving Sepsis Campaign: international guidelines for management of severe sepsis and septic shock: 2008. Intensive Care Med. 2008; 34: 17-60.

8. Sheffield JS. Sepsis and septic shock in pregnancy. Crit Care Clin. 2004; 20: 651-60.

9. OMS (Organização Mundial da Saúde). Educação para uma maternidade segura: módulos de educação. 2 ed; 2005.

10. Dare FO, Bako AU, Ezechi OC. Puerperal sepsis: a menores de óbitos por sepse puerperal tem sido descritas. 10,20 Por outro lado a mortalidade do grupo que desenvolveu choque séptico, coincide com os achados de outros autores. 21,22

Da mesma forma que as complicações, o uso de procedimentos foi mais freqüente neste grupo especifico de pacientes, quando comparando com a clientela habitual desta UTI obstétrica, ${ }^{11,16}$ o que pode ser justificado pela maior gravidade do grupo aqui estudado.

A abordagem cirúrgica do sitio de infecção, apesar de ser considerada fundamental no tratamento de pacientes com sepse puerperal genital,23 não tem sido considerada na prática por alguns, preferindose, às vezes, a manutenção da conduta conservadora.

Em suma apesar de se constituir em uma das maiores causas de morte materna no mundo,22 poucos estudos têm descrito esse grupo de mulheres. A presente investigação se reveste de importância na medida em que contribui para o acúmulo de conhecimento acerca do tema. Consideramos que estudar de forma detalhada este grupo de pacientes trouxe novas informações sobre a doença, dando maior suporte para a preparação dos centros a fim de lidar com a sepse puerperal de origem genital. preventable post-partum complication. Trop Doct. 1998; 28: $92-5$.

11. Amorim MMR, Katz L, Avil MB, Araujo DE, Valença M, Albuquerque CJM, Carvalho ARMR, Souza ASR. Perfil das admissões em uma unidade de terapia intensiva obstétrica em uma maternidade brasileira. Rev Bras Saúde Matern Infant. 2006; 6: 55-62.

12.González-rosales R, Ayala-leal I, Cerda-lópez Ja, Cerónsaldaña MA. Maternal mortality in the hospital general de matamoros Dr. Alfredo Pumarejo Lafaurie for a period of 10 years. Ginecol Obstet Mex. 2010; 78: 219-25.

13. Del Valle-Padilla MA, Ducoing-Díaz R, Tinoco-Jaramillo G, Campos-González R. That provides effective conditions for normal delivery, in order to reduce the number of Cesarean sections. Puerperal Infection. Analysis of 618 cases. Ginecol Obstet Mex. 1997; 65: 33-8.

14. Kankuri E, Kurki T, Carlson P, Hiilesmaa V. Incidence, treatment and outcome of peripartum sepsis.Acta Obstet Gynecol Scand. 2003; 82: 730-5.

15. Panchal S, Arria AM, Harris AP. Intensive care utilization during hospital admission for delivery: prevalence, risk factors, and outcomes in a statewide population. Anesthesiol. 2000; 92: 1537-44.

16. Amorim MMR, Katz L, Valença M, Araujo DE. Morbidade materna grave ou mortalidade materna near miss. Rev Assoc Med Bras. 2008; 54: 261-6. 
17. Kauss IA, Grion CM, Cardoso LT, Anami EH, Nunes LB, Ferreira GL, Matsuo T, Bonametti AM. The epidemiology of sepsis in a Brazilian teaching hospital. Braz J Infect Dis 2010; 14: 264-70.

18. Carvalho RH, Vieira JF, Gontijo Filho PP, Ribas RM. Sepsis, severe sepsis and septic shock: clinical, epidemiological and prognostic characteristics of patients in an intensive care unit in a university hospital. Rev Soc Bras Med Trop. 2010; 43: 591-3.

19. Castro EO, Figueiredo MR, Bortolotto L, Zugaib M. Sepsis and septic shock during pregnancy: clinical management Rev Bras Ginecol Obstet. 2008; 30: 631-8.

Recebido em 23 de setembro de 2010

Versão final apresentada em 12 de novembro de 2010

Aprovado em 30 de novembro de 2010
20. Dzhavakhadze MV, Daraselia MI . Mortality case analyses of obstetric-gynecologic sepsis. Georgian Med News. 2005; 127: 26-9.

21. Mabie WC, Barton JR, Sibai B. Septic shock in pregnancy. Obstet Gynecol. 1997; 90: 553-61.

22. Sereno Colo JA, Pineda Marquez JC. Septic shock in obstetrics. Ginecol Obstet Mex. 1990; 58: 338-45

23. Sauer I, Schröder W, Raumanns J, Rath W. Sepsis and SIRS (systemic inflammatory response syndrome) in the puerperium--pathogenesis and clinical management. Z Geburtshilfe Neonatol. 1998; 202: 30-4. 